\title{
OBJECTS AND OBSERVATIONS IN INFRARED ASTRONOMY
}

\author{
H. J. HABING \\ Sterrewacht Leiden, Leiden, The Netherlands
}

I have been asked to give you some astronomical arguments why coherent detection is a very valuable technique. With emphasis on the word 'some', I will try to fulfill this task. I will explain why I think that improved spatial and spectral resolution of infrared observations will be of great value for astronomical questions. In doing this I will concentrate especially, though not exclusively, on problems of star formation. The importance of these problems is reflected in the fact that a man like Immanuel Kant found them of sufficient fundamental interest to come up with some hypothesis about the formation of the solar system. Also the origin of life on the earth (and elsewhere in the universe) is tied in with stellar formation. In spite of its importance the subject has remained almost closed for systematic scientific research until the recent advance of infrared and millimeter observing techniques - that is, until the last 10 years.

The outline of this talk is as follows. First I discuss the success of coherent detection techniques in the radio range (Section 1), then a brief exposition will be given of the radiation mechanisms that produce significant infrared emission (Section 2). In Section 3 some objects are introduced that promise to be interesting and in Section 4 I give a summary.

\section{Coherent Detection in Astronomy: Some Results}

Probably the best argument in favour of coherent detection is to show you some results. In Figure 1a a map is presented of a source of radiation as obtained by Schraml and Mezger (1969) at $20000 \mu \mathrm{m}$ with a photometer in the focal plane of a diffraction limited single reflector of $43 \mathrm{~m}$ diameter. Figures $1 \mathrm{~b}$ and $1 \mathrm{c}$ show a part of Figure 1a mapped by bringing coherently together signals arriving in the foci of three $18 \mathrm{~m}$ reflectors placed at varying distances (up to $100000 \lambda$ ) from each other. The result was obtained at Cambridge by Wynn-Williams (1971). The increase in the details found is really astonishing! It could never have been obtained with a single dish instrument and coherent detection was the only way out. The last word at this moment is shown in Figure 1d, which is the same as Figure 1c, except that now also high spectral resolution has been obtained. It shows the distribution in the same area as in Figure 1c of the radiation emitted by highly excited neutral hydrogen, notably the line that results from the transition from level $n=110$ to $n=109$. The result has been obtained by Goss, Matthews, Sullivan and Wellington in much the same way as Wynn-Williams did, but with a system of 20 dishes, i.e. with more collecting power. This gave them a higher sensitivity and allowed them to use narrow filters to obtain the required frequency resolution. 


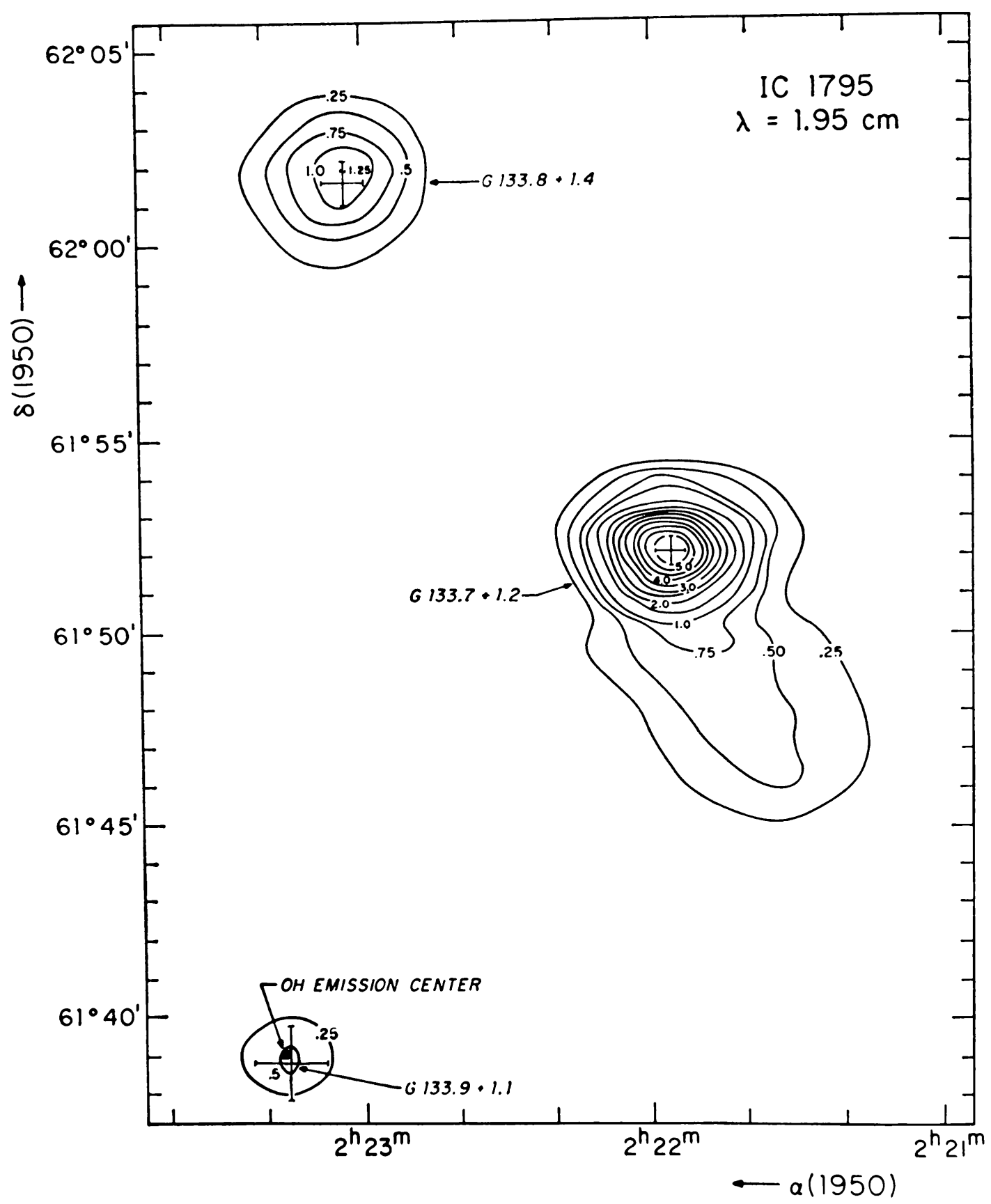

Fig. 1a. Isophotes of the continuum radiation at $\lambda=2 \mathrm{~cm}$ of the radiosource $\mathrm{W} 3$ adjacent to the $\mathrm{H}$ II region IC 1795. This figure represents the 'state of art' in radio astronomy in 1968. The map is due to Schraml and Mezger (1969).

From the analyses by the observers of the maps in Figure 1 it is clear that the progress in observing techniques led to significant discoveries. And it seems likely that this also will be the case for observations in the infrared wavelength range. Certainly, the skeptics among us may remark that the detection of more details does not always lead to deeper and better insight and that there are numerous examples, libraries full of astronomical observations, which have not contributed at all to the increase of 


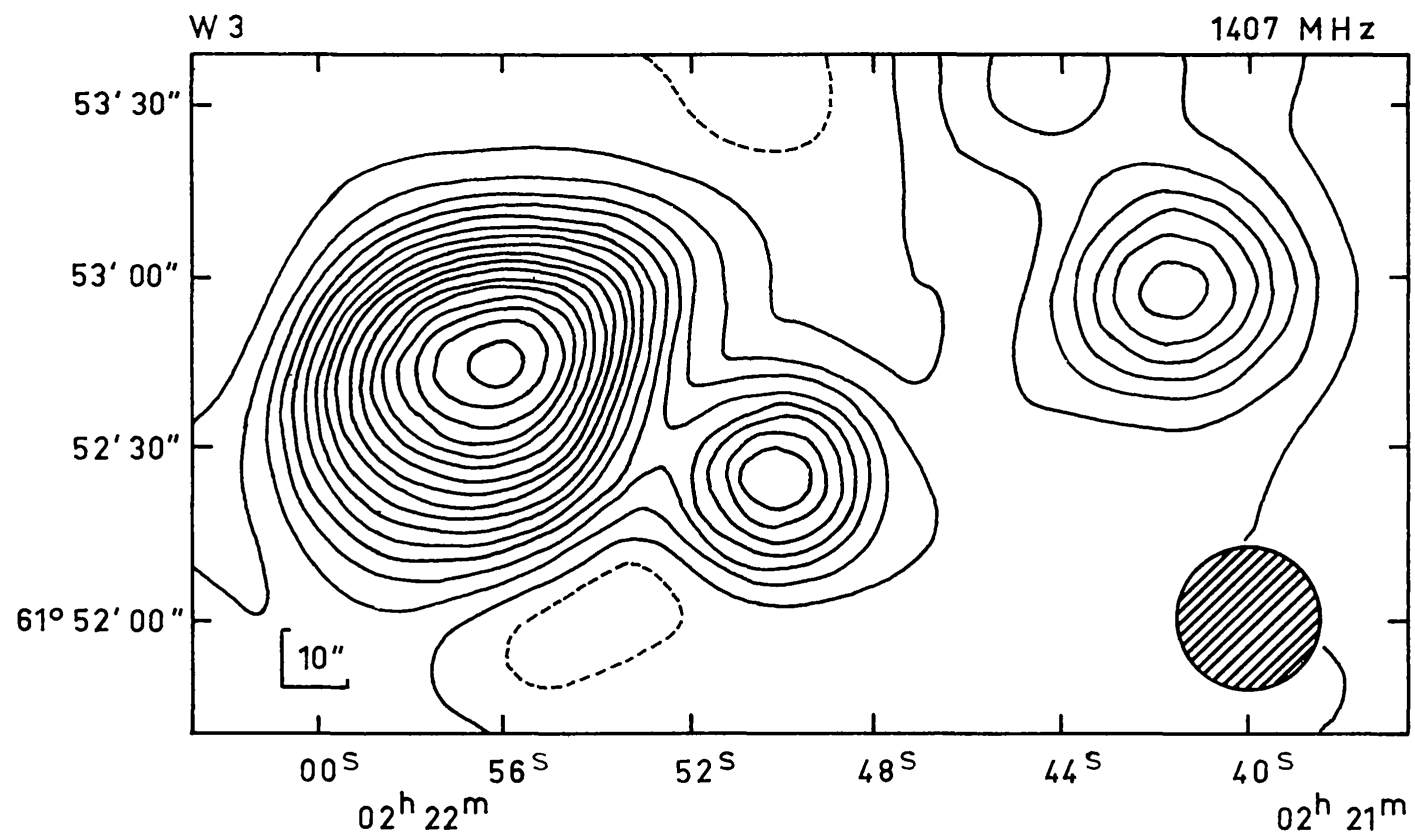

Fig. 1b

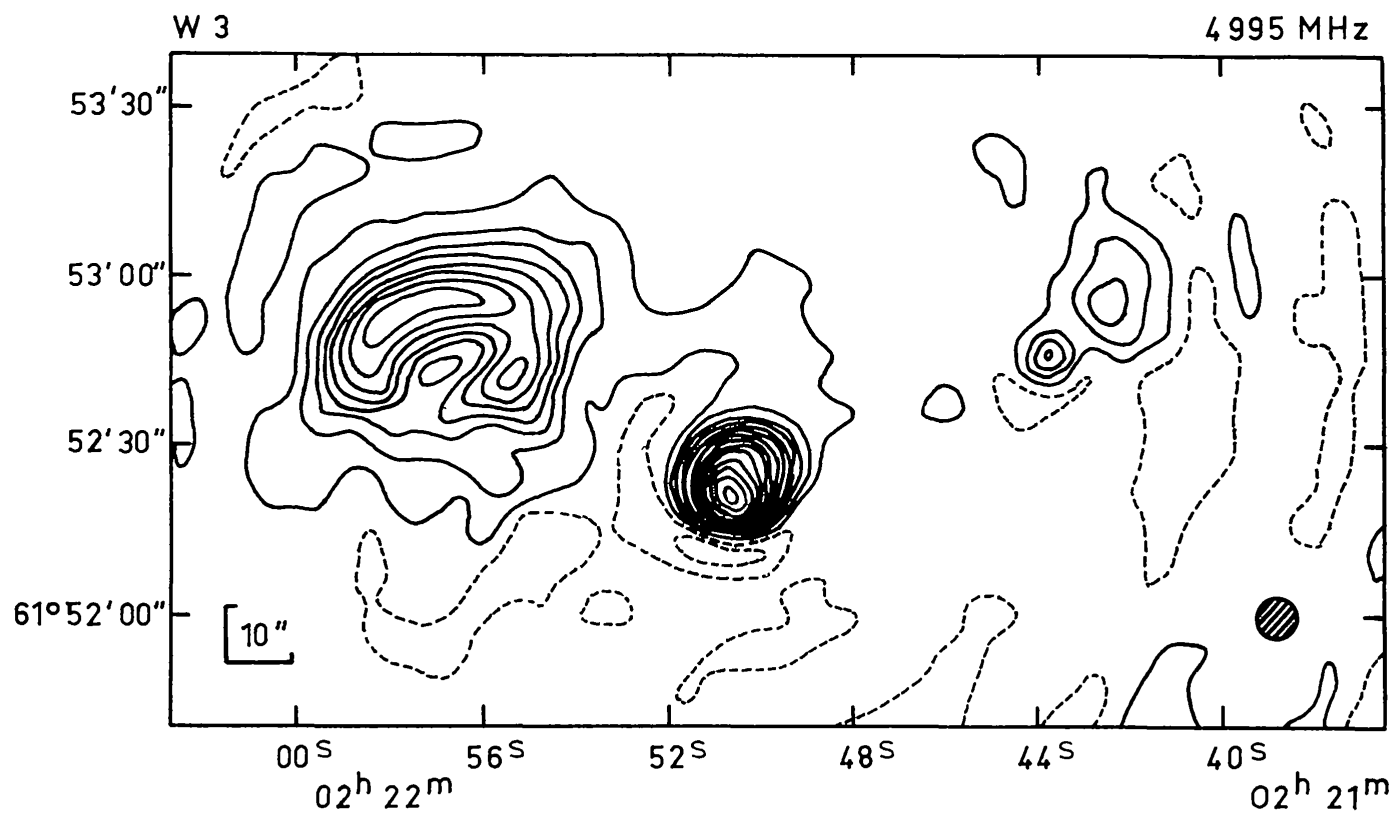

Fig. 1c.

Figs. $1 \mathrm{~b}-\mathrm{c}$. Isophotes of the continuum radiation at $\lambda=21 \mathrm{~cm}$ and $\lambda=6 \mathrm{~cm}$ of the radio source G 133.7+1.2, the brightest object in Figure 1a. This figure is the result of aperture synthesis observations based on a three element interferometer. The figures are due to Wynn-Williams (1971).

knowledge. While this is true, the recent experience of radio astronomy has shown that a totally new subject usually profits very much from improving observing techniques. It seems to me that infrared astronomy is still so young and so primitive that instruments can be improved without fear of producing uninteresting data. 


\section{W3 $H 109 \alpha$}

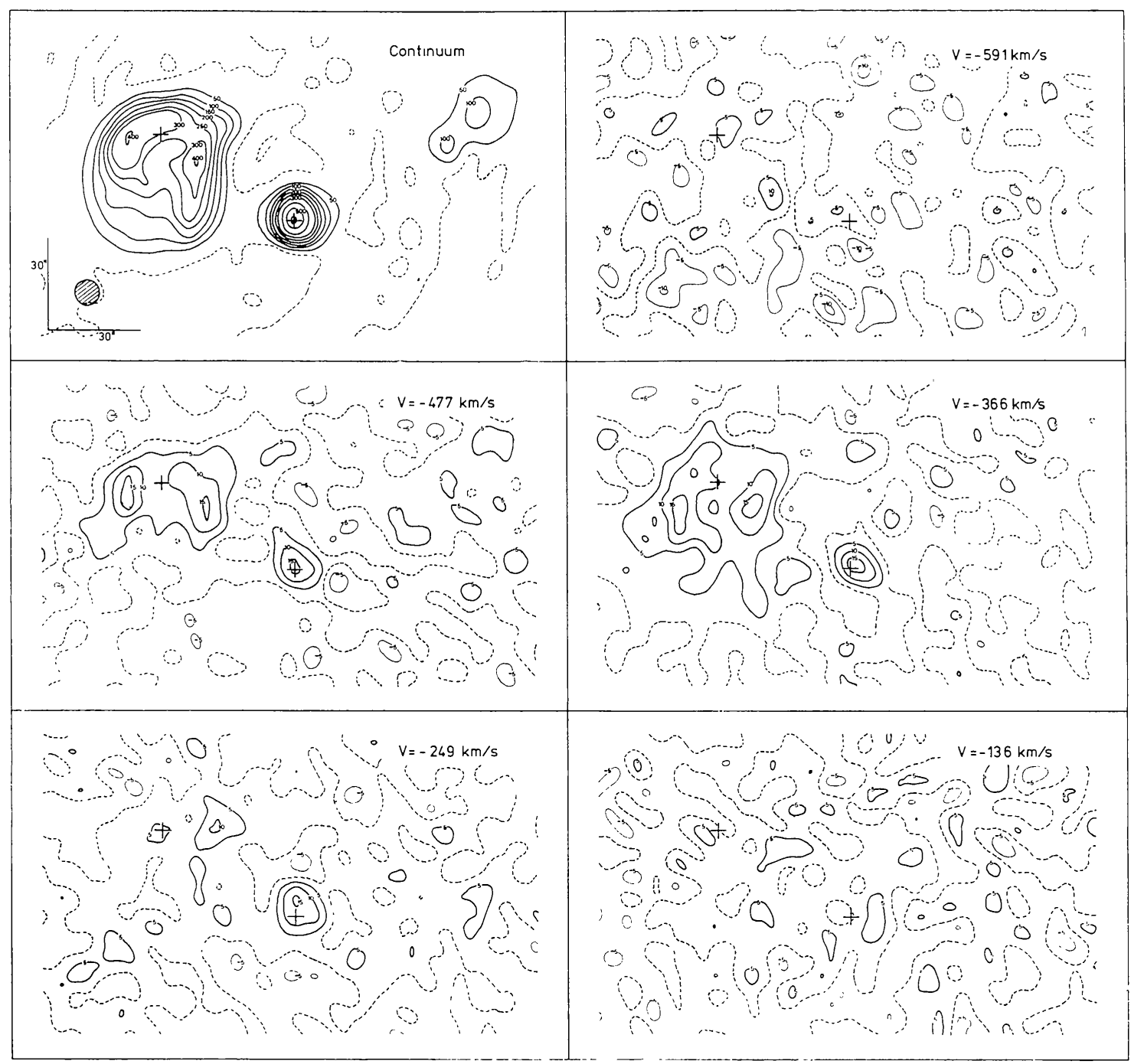

Fig. 1d. Isophotes of the radiation in a recombination line of hydrogen at $5009 \mathrm{MHz}$. The same object as in Figures $1 \mathrm{~b}$ and $\mathrm{c}$ is observed. Note the radial velocity structure of the source. The map was obtained in 1974 with a 20 element interferometer by W. M. Goss, H. E. Matthews, W. Sullivan and K. Wellington.

\section{Spectral Resolution: How is the Radiation Produced?}

\subsection{GeNERAL CONDITIONS}

In our Galaxy approximately $90 \%$ of the matter is inside stars. With some reservation in mind we can say that stars can be ignored as sources of infrared radiation.The $10 \%$ remaining matter, to be called for convenience, interstellar matter, is of direct interest for this talk. However, this unique name does by no means define unique physical conditions. Probably the most confusing fact about the interstellar medium is that it is so highly non-uniform. The density varies from 0.1 to $10^{6}$ atoms per $\mathrm{cm}^{3}$. The temperature, which by the way is defined by the Maxwell distribution of atoms and free 
electrons, varies from $5 \mathrm{~K}$ to $15000 \mathrm{~K}$. (Recent observations with the Copernicus satellite indicate, quite unexpectedly, that large portions of interstellar space may even be filled with a gas of density $10^{-3} \mathrm{~cm}^{-3}$ and a temperature of about $10^{6} \mathrm{~K}$.) A further factor of fundamental importance for the physical conditions of the gas is the ambient photon density. This density may be dominated by a single many star, but in most of interstellar space, say $90 \%$, the photons come from many stars in the background, so that there is no dominant single photon source.

It is customary, and physically significant to distinguish between circumstellar matter, within, say, $0.1 \mathrm{pc}$ from a star, and interstellar matter, further outside. It is an academic question whether or not circumstellar material belongs to the domain of stellar astronomy or of interstellar astronomy. Certainly circumstellar matter has a historical relation to the star - it may, for example, have been ejected from the star. But the physical processes occurring in circumstellar matter are more related to interstellar processes. Although, mass wise, circumstellar matter is unimportant it accounts for many of the infrared observations.

Another fundamental distinction is due to the physical state of the dominant constituent of all matter in the Galaxy: hydrogen. One distinguishes between three differrent sets of conditions. The distincion between regions with different sets of conditions is probably always rather sharp. (i) $\mathrm{H}_{2}$ regions, where the hydrogen is largely, and probably almost exclusively, in molecular form. This happens in cool $(T<50 \mathrm{~K})$ and dense ( $n>100 \mathrm{~cm}^{-3}$ ) clouds. (ii) $\mathrm{H}$ I regions, where the hydrogen is in atomic form. This is probably the case in most of interstellar space. Usually two conditions are distinguished: 'clouds', with $50<T<200 \mathrm{~K}, 2<n<300 \mathrm{~cm}^{-3}$, and the intercloud medium', $T>1000 \mathrm{~K}, n \simeq 0.2 \mathrm{~cm}^{-3}$. (iii) $\mathrm{H}$ II regions, where the hydrogen is almost completely ionized. Since hydrogen recombines fast compared to interstellar time scales, $\mathrm{H}$ II regions have to be kept ionized constantly. This is always being done by hard UV radiation from massive, hot stars ( $O$ and B type stars). Since massive stars have short lifetimes, they are found near their birth ground, the dark, cool, $\mathrm{H}_{2}$ clouds, and so are the $\mathrm{H}$ II regions. $\mathrm{H}$ II regions are usually rather dense $\left(n>100 \mathrm{~cm}^{-3}\right)$. The gas temperature in an $\mathrm{H}$ II region is determined by the radiation of the ionizing stars, and is rather close to the effective temperature of the stars, i.e. $T \simeq 10^{4} \mathrm{~K}$.

If hard UV photons can leak out of a dense cloud into the surrounding intercloud medium, relatively little radiation will be able to ionize large fractions of interstellar space. Whether this happens or not, is a 'hot' topic at this moment. The best evidence that it does happen comes probably from observations in other galaxies, notably in M 33.

Finally consider the composition of the interstellar medium. There is good observational evidence that about $90 \%$ of the atoms are hydrogen atoms, $10 \%$ are helium atoms, all the other elements constituting approximately $0.5 \%$ in number. Nevertheless the exact composition of the remaining elements is a major issue for investigation. Stars form out of the interstellar medium and ultimately they throw a considerable fraction of their mass back into the interstellar medium. During its stay inside the star, hydrogen is partially transformed by nucleogenesis in heavier elements and consequently the ejected gas is enriched in its trace elements. Hence one expects a conti- 
nuous enrichment of the trace elements during the history of the Galaxy. Unfortunately enrichment is a very slow process and difficult to follow observationally. Ironically enough, recent observations of the abundance of trace elements in the interstellar medium have shown that many elements are underabundant with respect to hydrogen, when their abundances are compared to those in the Sun. Since the Sun is an object of considerable age (say $5 \times 10^{9} \mathrm{yr}$ ), such a conclusion disagrees with the enrichment theory. 'Underabundance factors' range from 10 (nitrogen, for example) to 2000 (calcium and titanium). The standard explanation for this effect is that the trace atoms do exist, but are hidden in interstellar grains and that the discrepancy is only seemingly.

This leads us to the last, and most enigmatic constituent of the interstellar medium: the grains - small (diameter $<1 \mu$ ), solid particles of as yet unknown composition. The existence of the particles was first concluded in the late twenties when it could be proven that distant stars show extinction. Since the extinction is stronger in the blue than in the red, the effect is often called 'interstellar reddening'. Very extensive theoretical and observational work has been done on deriving the properties of the solid particles from the precise wavelength dependence of the extinction. Various grain types have been proposed: ice particles, graphite particles. But it never became possible to make a choice between the grain models. This situation has changed in the last few years because of at least two different break throughs. The first break through is the detection of various bands in the extinction curve. The existence of such bands has been known for more than 30 years in the optical wavelength range; the best known of these is a wide band near $4430 \AA$, for which, however, no explanation exists. Now satellite observations in the last few years have shown an absorption band of approximately $500 \AA$ wide near $2200 \AA$, usually attributed to graphite grains, whereas infrared observations showed the existence of two absorption bands, one at $3.1 \mu$, probably due to $\mathrm{H}_{2} \mathrm{O}$-ice, and one at $9.7 \mu$, probably produced by a silicate type material. Whether these various materials are united in the grains or whether several types of grains exist simultaneously, is still an open question. It is conceivable that more bands like the 3.1 and $9.7 \mu$ bands will be found at other wavelengths in the infrared. This would require at least a spectral resolution $\Delta \lambda / \lambda<10^{-1}$, and heterodyne techniques may not be necessary in first instance. The significance of such a discovery is obvious: they will help to find the nature of the grains.

The second breakthrough in our understanding of the grains may be in the underabundance of the trace elements in the interstellar medium. At the moment it appears that the trace elements can be devided into two groups: those with only a mild underabundance (by factors between 2 to 50 ) and those with very strong underabundance (by factors between 100 and 2000). The latter group contains Ca, Ti, A1. G. B. Field has noted another characteristic of the last three elements, which makes them differ from the others: In a cooling gas mixture they are the first to disappear because of solidification processes. The implications of this are not yet fully clear, but it indicates that the interstellar abundances may give hints on the actual grain formation processes. Solidification probably occurs in very extended, cool circumstellar shells around stars in very late evolution stages or around stars in very early evolution stages. 


\subsection{Thermal RADIATION BY DUST PARTICLES}

Up until now most of the observations in the infrared concern thermal radiation from grains. The grains constantly absorb photons from the incident flux, and radiate the energy away at other wavelengths, in agreement with their emission properties. Theoretical calculations indicate that grain temperatures lie between 10 and $50 \mathrm{~K}_{\text {in }} \mathrm{H}_{2}$ and in $\mathrm{H}$ I regions. However, in $\mathrm{H}$ II regions and in circumstellar envelopes the grain temperature may go up to a few hundred $\mathrm{K}$. It is somewhat surprising that grains can endure the harsh conditions in $\mathrm{H}$ II regions. Both the hard UV photon flux and the corroding collisions with energetic electrons and protons tend to destruct the grain. But whatever the explanation is, observations tell us that grains do exist in $\mathrm{H}$ II regions.

From the mentioned temperatures it is immediately clear that the grain emission is mainly in the infrared. It is also obvious that high spectral resolution is not required to study this type of radiation. Only moderate resolution, say $10^{-3}<\Delta \lambda / \lambda<10^{-2}$, will be enough to find and to study the specific absorption bands, and a resolution of $\Delta \lambda / \lambda \simeq$ $\simeq 1 / 50$ is sufficient to study the dependence of the emissivity on wavelength, which may turn out to be an interesting fingerprint of the grain material. However, to obtain a clean fingerprint, high spatial resolution will be required in order to study the distribution of the infrared emission at various wavelengths and to separate contributions to the infrared flux from regions with very different physical conditions, for example the inner part of an $\mathrm{H}$ II region from a cool outside layer of neutral hydrogen.

\subsection{Molecular Lines}

From the point of view of molecular spectroscopy the infrared is a very rich spectral region. Still, infrared astronomy has hardly contributed to molecular astrophysics. Molecular lines have been found at $\lambda<10 \mu \mathrm{m}$ in bright cool stars, but interstellar lines have not yet been seen, probably because of lack of detector sensitivity, poor transparency of the atmospheres and the absence of high spectral resolution. From the observation of many interstellar lines in the centimeter and millimeter range and from electronic transition lines in the UV we know that interstellar molecules abound, and the infrared window should be very rich indeed. However, there is a clear lack of predicted lines and line strengths. Recently a few groups have attempted to make such predictions: for CO see Goldreich and Kwan(1974), and De Jonget al.(1974); for $\mathrm{HCl}$ see Dalgarno et al. (1974). Very promising are also lines of $\mathrm{HD}$, the strongest one $(\mathrm{J}=1 \rightarrow 0)$ is at $112 \mu$. Interesting, but very difficult to detect appears to be the $28 \mu$ line of $\mathrm{H}_{2}$.

Let me describe briefly why astronomers appreciate lines so much. Line measurements yield information about velocities and level populations. In principle, given sufficient lines (and sufficient transparency of the object!) one can deduce both the dynamical status of the object under consideration and the physical conditions (temperatures and densities). However, this process of deductive reasoning is never simple and straightforward and the more lines one measures and the more accurate the line profiles are known, the better. This provides an answer to an often asked question: since one can do molecular astrophysics in the $\mathrm{mm}$ and $\mathrm{cm}$ range from the ground, why 
should one work toward difficult experiments, preferentially from satellites, in the infrared? The obvious answer is: the more molecules and the more lines one observes, the easier it will be to understand the conditions in the emitting regions. Molecules like $\mathrm{CH}_{4}, \mathrm{HCl}$ (and other hydrides) and $\mathrm{HD}$ will probably turn out to be very important building stones in our knowledge of how dark clouds give birth to stars. They can only be seen in the infrared. And our only chance to observe the most abundant molecule $\left(\mathrm{H}_{2}\right)$ in emission, is around $28 \mu$.

\subsection{ATOMIC AND IONIC LINE EMISSION}

In interstellar space atoms and ions are usually in their lowest energy levels. Generally the densities are too low to populate the higher levels considerably. However, a significant number of ions may populate the multiplet levels of the lowest electronic configuration. For example the $1 s^{2} 2 s^{2} 2 p^{2}$ configuration of $\mathrm{C}^{0}$ and $\mathrm{O}^{++}$and the $1 s^{2} 2 s^{2} 2 p^{4}$ configuration of $\mathrm{O}^{0}$ have five energy levels, the lowest three constituting a ${ }^{3} P$ level, the next an ${ }^{1} D$ and the highest an ${ }^{1} S$. For $C^{0}$ the levels are at $16.4,43.5,10194,21648 \mathrm{~cm}^{-1}$ from the ground level, for $\mathrm{O}^{++}$at $113.4,306.8,20271,43184 \mathrm{~cm}^{-1}$. Transitions among the lowest levels, belonging to ${ }^{3} P$, give therefore rise to infrared emission lines; transitions between ${ }^{1} S$ and ${ }^{1} D$, and between these and ${ }^{3} P$ give rise to visible emission lines. The visible lines of $\mathrm{O}^{++}$have been studied very well over the last fourty years. For lines to be in the visible part of the spectrum, it is obvious that hot regions are required, which implies in practice $\mathrm{H}$ II regions. This, of course, is not required for the excitation of the ${ }^{3} P$ levels, that produce only infrared lines. However, cool regions will be always $\mathrm{H}$ I regions and therefore one does not expect $\mathrm{O}^{++}$to be present. Infrared lines from $\mathrm{H}$ I regions will be expected only from atoms and ions with low ionization potentials such as $\mathrm{C}^{0}, \mathrm{C}^{+}, \mathrm{N}^{0}, \mathrm{O}^{0}$.

Fine structure lines have not yet been observed in the infrared, except for a $\mathrm{Ne}^{+}$line at $12.8 \mu$, which has been seen in a few very dense circumstellar $\mathrm{H}$ II regions. Many $\mathrm{H}$ II regions are made invisible by intervening dust and have been detected through their radio emission. Quite often the $\mathrm{H}$ II regions have very high surface brigtness and are probably young and in an active stage of evolution. Infrared lines may pass through the dust layers and allow us a closer look at the inner conditions. Lines produced in $\mathrm{H}$ I regions have never been detected, with the $21 \mathrm{~cm}$ line as a great exception. Detection of such lines (e.g. the [C II] line at $156 \mu$ ) would be of great help in establishing the physical conditions inside $\mathrm{H}$ I regions, e.g. temperature and density. Such observations would supplement in a splendid way the recent measurements of interstellar absorption lines from the different ground levels, that are being made by the Copernicus satellite.

Theoretical predictions of linestrengths for interstellar lines have been made for H I regions by Pottasch (1973) and for H II regions by Petrosian (1970).

\subsection{NON-THERMAL EMISSION PROCESSES}

Non-thermal processes account for most of the extraterrestrial radio radiation. By extrapolation one could expect, a priori, to find non-thermal infrared emission. An 
object, which certainly will emit such radiation, is the Crab nebula, because the visible and the radio spectra are both synchrotron emission from the same volume. Still, there is a basic limitation for the occurrence of non-thermal infrared emission, because such emission requires large energy production rates. For example, a relativistic electron emitting mainly at $100 \mu$ has a lifetime 40 times shorter than one that emits mainly at $20 \mathrm{~cm}$, but still it will emit in total 40 times more energy. Infrared synchrotron radiation will therefore be restricted close to regions of particle acceleration. Presumably the same holds for other possible non-thermal effects. Quasars, Seyfert galaxies, galactic nuclei and pulsars are of course the prime objects to look for nonthermal infrared emission. From an observational point of view the important quantities to observe are sizes, frequency dependence and polarization. Especially for significant size measurements resolutions of $1^{\prime \prime}$ or less in the infrared are required. To study frequency resolution and polarization properties, coherent detection techniques are probably not required.

No predictions exist, other than wild speculations, about the expected non-thermal infrared fluxes. Let us consider one observational example. The Seyfert Galaxy NGC 1068 has a maximum flux at $20 \mu$ of about 6 flux units $\left(=6 \times 10^{-26} \mathrm{Wm}^{-2} \mathrm{~Hz}^{-1}\right)$. Polarization studies (Knacke and Capps, 1974) suggest that a significant fraction of this flux $(10,20 \%$ ? ) may be non-thermal.

\section{What Objects to Look at?}

After having discussed what processes and constituents produce the infrared photons it is necessary to consider briefly the objects which have been detected or will be detected. Such considerations will put an emphasis on spatial resolution - another potentiality of coherent detection.

(i) Let me first mention, in passing, the Solar System. The planets have been studied extensively in the red and near infrared, and certainly are prime objects in the far infrared (say $\lambda>20 \mu$ ). At closest approach Jupiter has a diameter of some 50", Uranus of $4^{\prime \prime}$, and Venus of some 60". Detailed mapping of the planets will no doubt turn out to be important - provided the mapping will not have been done earlier by space vehicles. Other objects that, no doubt, will be important to study are the comets.

(ii) Starting now from the other end, let us speculate for a second on infrared emission from the Universe. Of prime importance is, of course, the measurement of the $3 \mathrm{~K}$ background and its isotropy. However, I would be much surprised if coherent detection techniques should be of any use in such a measurement. The $3 \mathrm{~K}$ background has probably very little spatial and spectral resolution, and, since its detection requires absolute measurements, a different technological approach appears required. This statement is based on the radio-experiments: the background has been detected at centimeter wavelengths with a very simple, but extremely well-calibrated receiving system.

But the universe contains small scale structures: the galaxies. These provide very rich, though still virginal hunting grounds for high resolution detection devices. Only 
a few galaxies have been found at $\lambda>30 \mu \mathrm{m}$ : in the literature I know of positive detections at $\lambda=350 \mu \mathrm{m}$ only for M82 and NGC253. At $\lambda \lesssim 20 \mu \mathrm{m}$ several galaxies have been studied. Consider for example NGC 253, which has been observed at five wavelengths between 1.6 and $20 \mu \mathrm{m}$ by Becklin et al. (1974) through various diaphragms. The conclusion is that the observed infrared flux (total flux density observed at 10.1 $\mu \mathrm{m}$ is 10 flux units) is strongly concentrated towards the nucleus and that approximately $50 \%$ of this flux density comes from a core of $150 \mathrm{pc}$ diameter The core coincides accurately with a non-thermal radiosource of similar diameter. This coincidence points to what is probably the first major goal in extragalactic infrared atronomy: the study of galactic nuclei. Astronomically speaking this is a hot topic. Radioastronomical observations have shown that galactic nuclei are responsible for much violent action in galaxies. There appears to be a progression beginning with quiet galaxies like the Andromeda Nebula (M 31), and with quasars at the other extreme end. Somewhere in this range fall the Seyfert galaxies - small objects with violently active nuclei, one of which was already mentioned in section 2.5: NGC 1068. Rather convincing evidence exists that galaxies with nuclei active at radio wavelengths also have strong infrared emission from the core. The observed relation, however, also indicates that radio and infrared emission originate in different processes. The radio emission is synchrotron emission from particles presumably accelerated in the galactic nuclei, whereas the infrared emission apparently comes from dust radiation.

(iii) The nucleus of our Galaxy has been recently an active source of energy, although, probably, at a rather low level when compared to several other galaxies. This is shown mainly by radio observations. Actually the center of the Galaxy was one of the very first sources detected in the radiosky, just before World War II. A recent discussion of radio and infrared properties has been given by Mezger (1974) and I summarize briefly his conclusion. As shown by Figure $2 \mathrm{a}$ several components are to be distinguished, among them the so called nuclear disk. In the center of the disk there is an extended, low density $\mathrm{H}$ II region which is shown again in Figure $2 \mathrm{~b}$ in more detail. Several discrete sources are noticeable inside the extended component, all of which appear to be thermal, except one. The central source in Figure 2b, called Sgr A, contains two small components: 'Sgr A West' and 'Sgr A East'. Sgr A East is the one non-thermal source in Figure $2 \mathrm{~b}$, with a diameter of approximately $7 \mathrm{pc}$, whereas Sgr A West probably is a dense $\mathrm{H}$ II region of approximately $2 \times 2 \mathrm{pc}$. However, observations of hydrogen recombination lines have shown that the gas in Sgr A West has high speed motions - up to $200 \mathrm{~km} \mathrm{~s}^{-1}$. Sgr A West is also a small strong infrared source as measured by Borgman et al. (1974; 300 flux units at $10 \mu \mathrm{m})$. It certainly is an interesting object (see Figure 2c).

One may expect (and the limited experience has confirmed this so far) that the infrared picture is as complex as the radio picture - implying that detailed mapping at various wavelengths may be required before firm and significant conclusion can be drawn. Since the infrared radiation appears to be thermal radiation by dust, no sharp spectral features are expected. However, molecular lines have been found in the millimeter wavelength range and infrared molecular lines may also be present. 

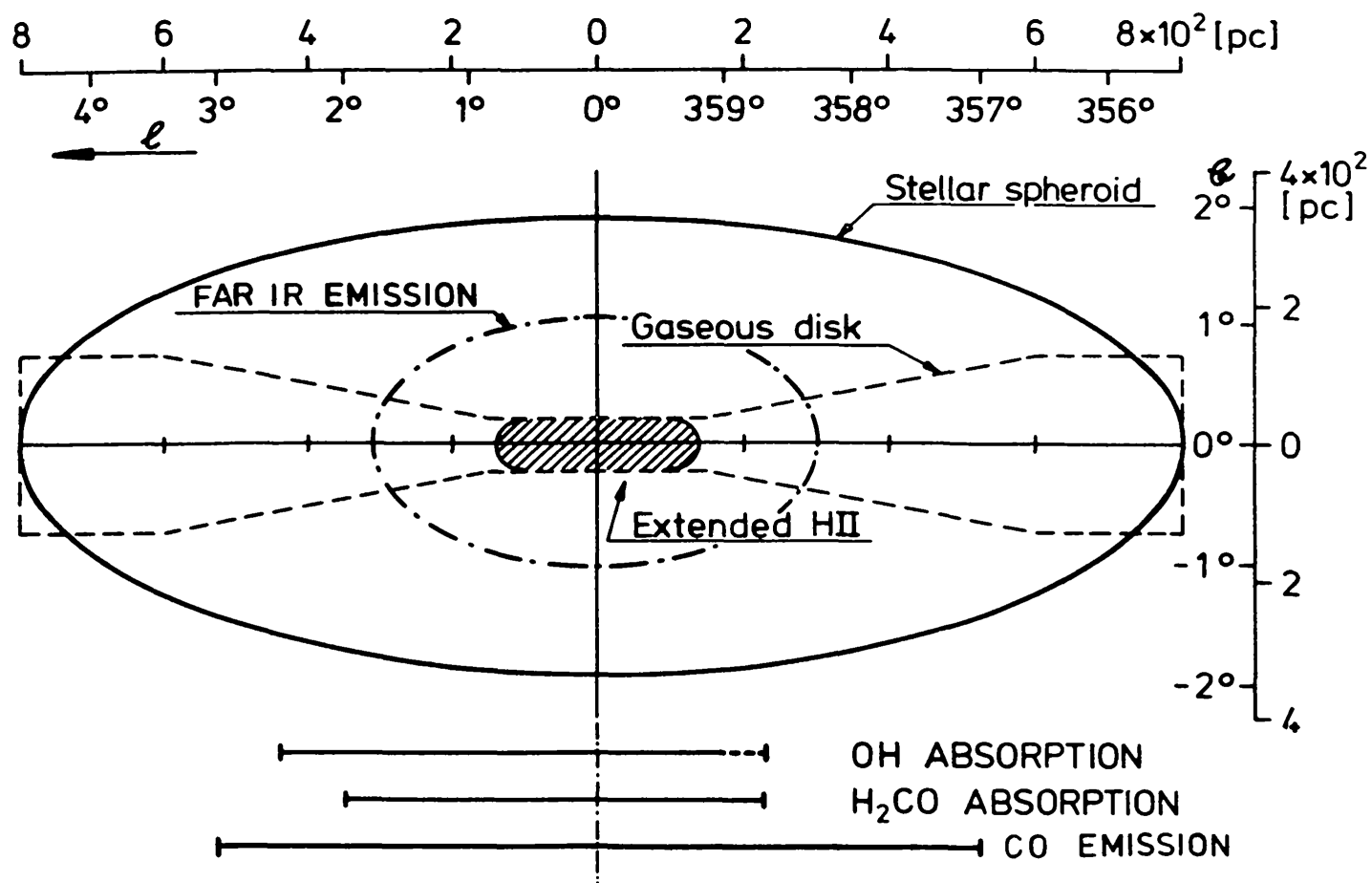

Fig. 2a. Schematic outline of the main components in the galatic nucleus. The figure is due to Meager (1974).

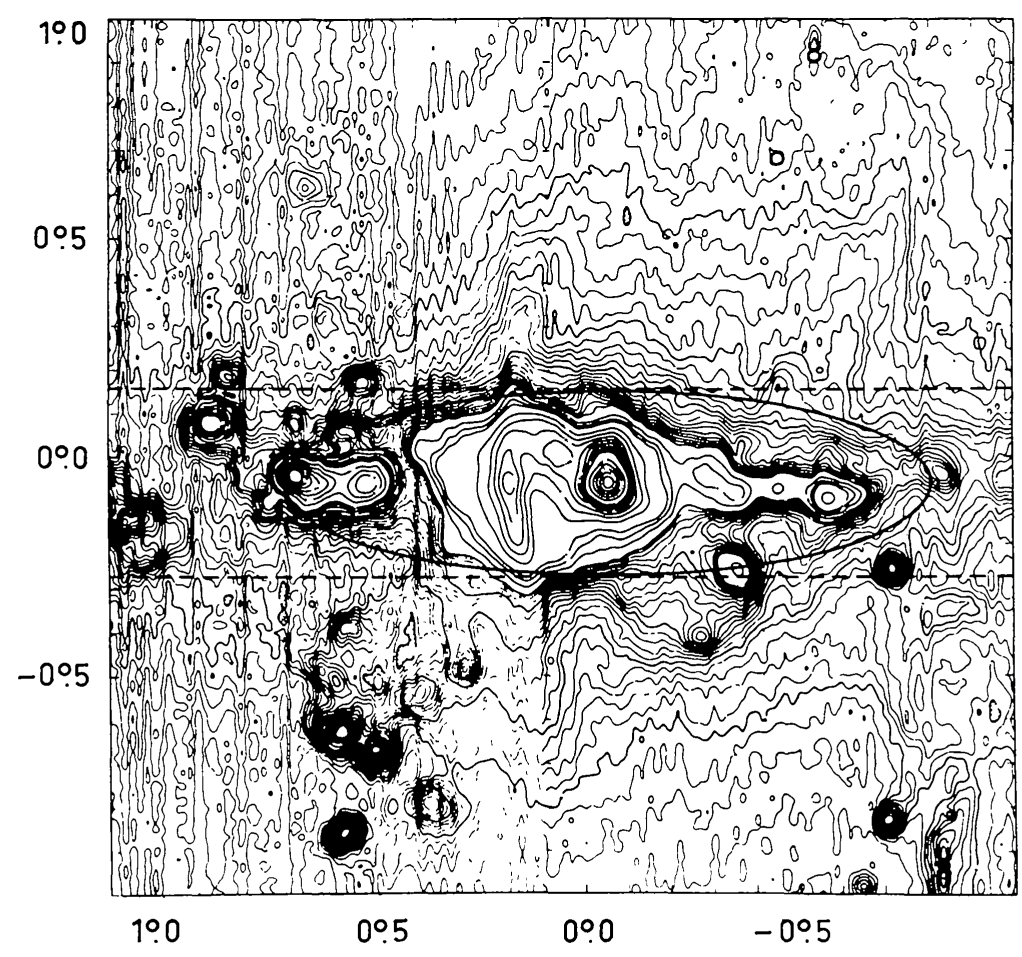

Fig. 2b. Isophotes of the continuum radiation at $6 \mathrm{~cm}$ from the galactic center. The map is due to Altenhoff, Downes, Pauls and Schraml as presented by Mezger (1974). The full curve corresponds to the hatched area in Figure 2a called 'Extended H II'. The strong source at the center is Sgr A. More detailed mapping shows Sgr A to consist of two components: Sgr A East and Sgr A West. 


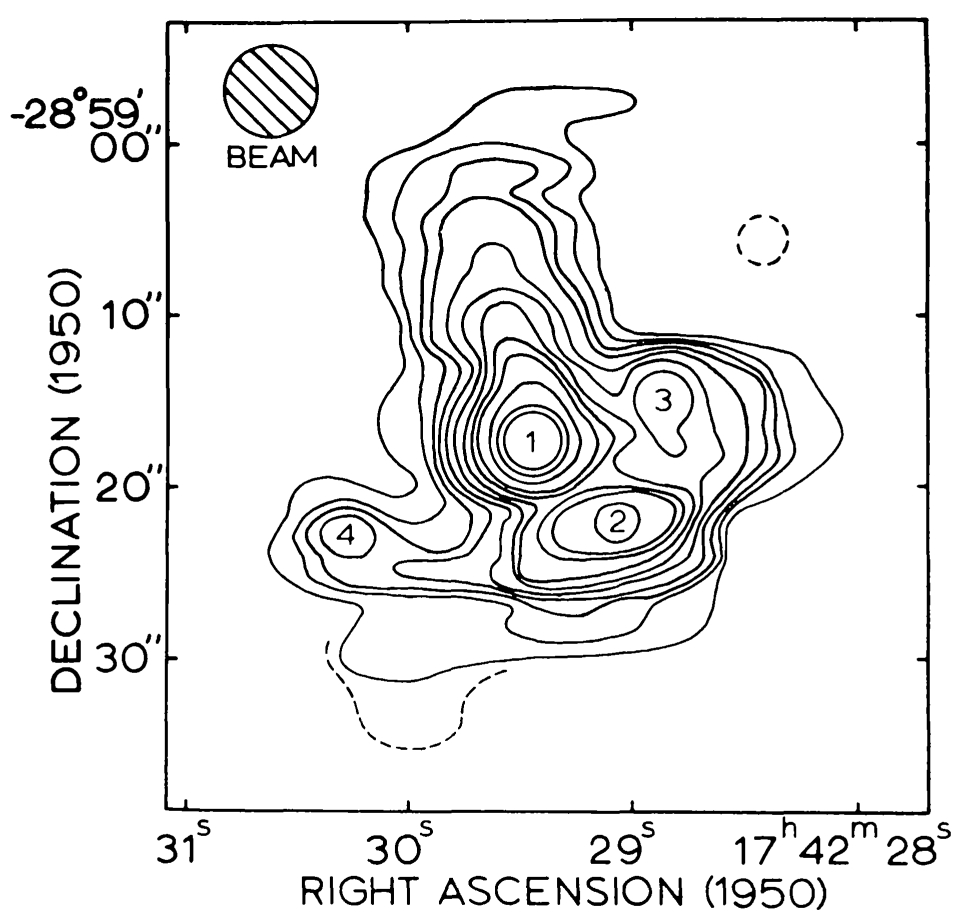

Fig. 2c. Isophotes of radiation at $10.5 \mu \mathrm{m}$ at the galactic center, as given by Rieke and Low (1974). The position of source 1 has been remeasured by Borgman (1974) and appears to coincide with Sgr A West.

(iv) Consider now infrared radiation associated with stars. In the beginning of this section I said that stars are not important infrared emitters and, in fact, the infrared emission we see from stars probably comes from circumstellar shells at considerable distances from the stellar photosphere. To show that stellar photospheres will not produce much infrared radiation consider a star of magnitude $V=+2.0$, which means a flux density of 670 flux units at $5480 \AA$ or at $5.474 \times 10^{14} \mathrm{~Hz}$. In the sky there are only some 50 stars up to that magnitude. The coolest among them are early M stars (M1M3), with color temperatures between 3500 and $3800 \mathrm{~K}$. This means that the flux density at $20 \mu \mathrm{m}$ due to these stars will be 110 flux units - a measurable, but not very strong source. Hotter stars of the same magnitude will necessarily be weaker, and hence photospheres of main sequence stars will produce very few observable infrared sources. Stars with significant flux densities at $\lambda>10 \mu \mathrm{m}$ will be objects with very extended atmospheres, or, rather, circumstellar shells. These may be cool and radiate through dust grains ( $M$ giants and supergiants) or they may be hot and ionized and radiate by the bremsstrahlung process (WR stars).

The astrophysical significance of infrared (circum)stellar observations goes into two directions: towards the study of late stages of evolution and towards that of early stages. Both stages are very poorly understood. During the last fifty years reasonably good insight has been obtained about the intermediate stage of stellar evolution (main-sequence stage), when the star is a rather well isolated, dynamically quasistationary object. However, during the formation and during the decay (except when the decay is explosive) the star is wrapped in an extensive, cool layer, so that infrared 
observations will be very useful, if not essential, for our understanding of the gasdynamics and other basic physical processes in the atmosphere. Part of the infrared emission will be reradiation by grains, giving an essentially featureless continuum, except for broad bands, which probably will not yield any dynamical information and only very limited other phsysical information. But molecular lines will also be present (in absorption and in emission) and may, ultimately, teach us about the structure and dynamics of the circumstellar envelopes.

Let me describe, at this point, a currently rather popular subject: cocoonstars. Numerical calculations have shown conclusively that in a gravitationally collapsing cloud a stellar nucleus will form rather quickly: the collapse time at the center of the cloud is much shorter than in the outer parts. Recently Kahn has argued that especially during the formation of massive stars the collapse time of the outer layers is so long compared to the contraction time of the core that the central object will develop quickly into a main sequence star, which then will grow by accretion and, gradually, will climb up the main sequence. During the accretion the high luminosity of the star will give rise to radiation pressure effects in the collapsing cloud, leading to the formation of a very dense, very hot circumstellar shell in which the dust temperature rises to the melting temperature of, say, $3000 \mathrm{~K}$. Such circumstellar shells will be strong infrared emitters. In fact, they may have been discovered already. The Becklin-Neugebauer star in the Orion nebula may be a prime example, but other suspects exist, e.g. near the $\mathrm{H}$ II region DR 21. Accurate flux-densities of such objects through the whole infrared range and the detection of molecular lines would be very valuable and may open further an already very promising field of theoretical and observational research. As a final comment, let us consider the possibility of coherent interferometry in the infrared. Cocoons, such as just described, or circumstellar envelopes, such as have been observed spectroscopically, will have sizes of, say, $10^{3}$ to $10^{5}$ solar radii. Since the Sun has an angular diameter of $0.5^{\circ}$ at its distance of $1 \mathrm{AU}$, circumstellar envelopes will have an angular diameter of 1 arcsecond at distances between 10 and $100 \mathrm{pc}$. If we could do aperture synthesis in the infrared with only a tenfold improvement in resolution compared to what is being done at $6 \mathrm{~cm}$ (see Figures $1 \mathrm{~b}$ and 1c), we will be able to measure directly the sizes of the circumstellar envelopes!

(v) Finally there are the $\mathrm{H}$ II regions, certainly the best studied infrared emitters. At $\lambda<20 \mu \mathrm{m}$ several $\mathrm{H}$ II regions have been mapped with a resolution of the order of a few arc seconds. The results are quite spectacular, especially when they are compared with radio observations (Figure 3). Nevertheless the implications of the observations are not yet fully understood. Most of the infrared emission at $\lambda<20 \mu \mathrm{m}$ comes from the region of ionized gas, but must be due to hot dust grains mixed with the ionized gas. Of immediate importance is the question what the properties of these grains are. Are they smaller in size and fewer in (relative) abundance, and are they different in constitution? Such effects can be expected when the grains are exposed to the erosion processes by ultraviolet photons and hot electrons. More observations with arcsecond resolution at various wavelengths between 10 and $70 \mu \mathrm{m}$ appear required. At $\lambda \gtrsim$ $\gtrsim 100 \mu \mathrm{m}$ the emission may come from dense neutral material around the $\mathrm{H}$ II region. 


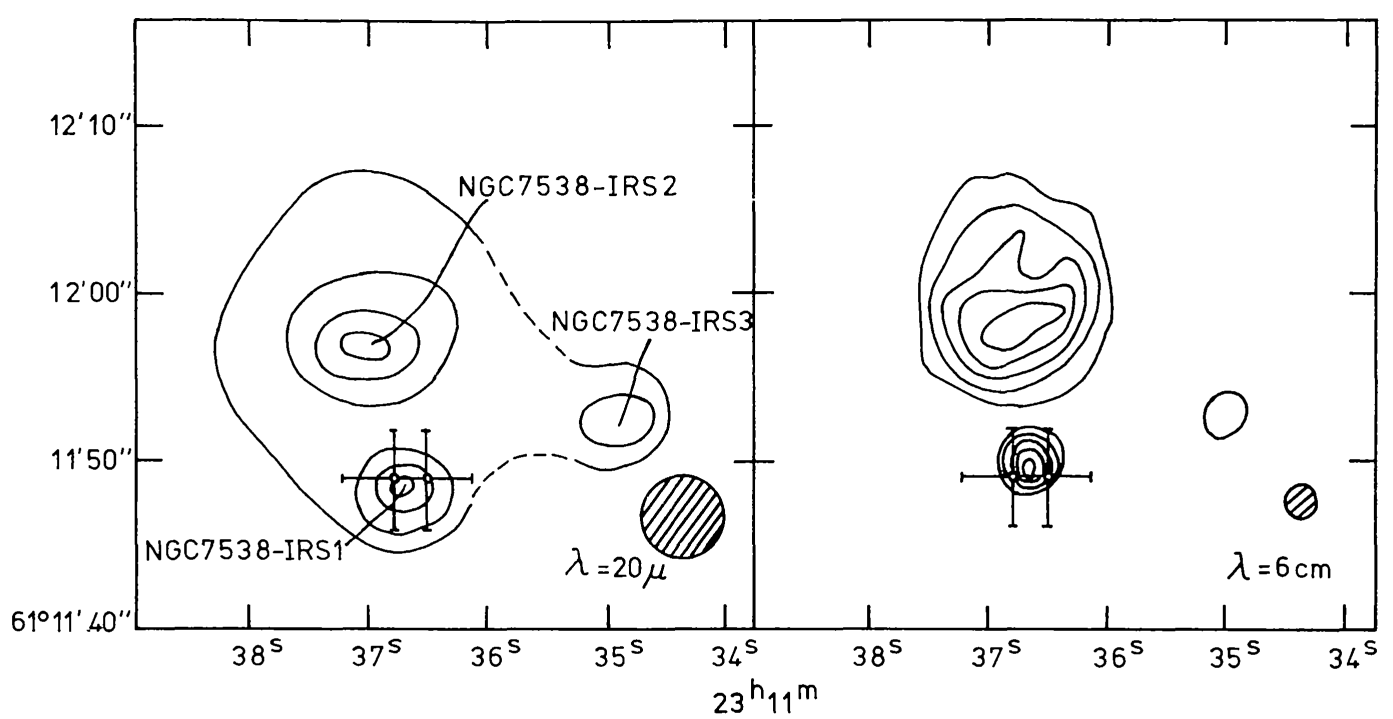

Fig. 3. (Left) Isophotes of the $20 \mu \mathrm{m}$ continuum radiation from a small radiosource near the $\mathrm{H} \mathrm{II}$ region NGC 7538. The map has been obtained by Wynn-Williams et al. (1974). Very likely the radiation is thermal emission by hot dust grains. (Right) Isophotes of the $6 \mathrm{~cm}$ continuum radiation from the same radiosource. The map has been obtained by Martin (1974). The radiation is bremsstrahlung from the ionized gas. The two crosses indicate the position of a source emitting strongly masered $\mathrm{OH}$ lines. This maser is probably located in the very dense shell surrounding the $\mathrm{H}$ II region that is a left over from the time of star formation.

This possibility can be checked when sufficient spatial resolution comes available, of say $10^{\prime \prime}$ at $100 \mu \mathrm{m}$.

An other interesting future experiment will be to detect ionic fine structure lines from $\mathrm{H}$ II regions, as has been described in Section 2.4 .

The overall significance of infrared observations of $\mathrm{H}$ II regions is that they clarify the events associated with the birth of massive stars. In addition they may help us to answer some crucial questions about the nature of the dust grains.

\section{Summary}

At this point I expect you, Experimentors, to start smiling friendly but depreciatingly for my emphasis on high spatial resolution, as if infrared aperture synthesis work were anywhere within reach. I will therefore stop and avoid further irritation by summarizing my main points.

At $\lambda<20 \mu$ m many good results have been obtained: galaxies, the galactic nucleus, circumstellar shells, $\mathrm{H}$ II regions and protostars have been observed. A larger sample of objects is desired, some improvement in spatial resolution (the more the better, but the state of art is good enough for the moment) and more sensitivity. But at $\lambda>20 \mu \mathrm{m}$ the results are less spectacular. Spectral resolution hardly exists, although moderate resolution, $\Delta \lambda / \lambda<10^{-2}$, will be important for the detection of absorption bands. Very high spectral resolution, $\Delta \lambda / \lambda<10^{-4}$ to $10^{-5}$, will be required for the detection of lines, a prime object for coherent detection, once it becomes operational. The lines 
may be molecular lines and fine structure ionic lines. Collapsing dark clouds, masers and protostars will then be among the first objects to observe - and the observation will be rewarding. If aperture synthesis mapping in the infrared becomes possible, if this ever happens, then again a wide field opens: detailed study of galactic nuclei in the far infrared will yield rich information on where dust formation processes take place, and small scale phenomena near protostars will tell us how the early collapse phase develops and how cocoons look like.

The final search is for increasing sensitivity: more objects and faster measurements will enlargen our sample and broaden our views. A challenging future lies in front of us!

\section{References}

Becklin, E., Fomalont, E. B., and Neugebauer, G.: 1973, Astrophys. J. 181, L27.

Borgman, J., Koornneef, J., and De Vries, M.: 1974, Proceedings Eighth ESLAB Symposium, Frascati, June 1974 (ed. by A. Moorwood).

Dalgarno, A., De Jong, T., Oppenheimer, M., and Black, J. H. : 1974, Astrophys. J. 192 , L37.

Goldreich, P. and Kwan, J.: 1974, Astrophys. J. 189, 441.

Jong, T. de, Chu Shih-I, and Dalgarno, A.: 1974, Center for Astrophysics preprint, No. 156.

Knacke, R. F. and Capps, R. W. : 1974, Astrophys. J. 192, L19.

Mezger, P. G.: 1974, Proceedings Conference on Research Programmes for the New Large Telescopes (ed. by A. Reiz)

Petrosian, V.: 1970, Astrophys. J.159, 833.

Pottasch, S. R.: 1973, Astron. Astrophys. 24, 305.

Rieke, G. H. and Low, F. J.: 1974, Astrophys. J. 184, 415.

Schraml, J. and Mezger, P. G.: 1969, Astrophys. J.156, 269.

Wynn-Williams, C. G.: 1971, Monthly Notices Roy. Astron. Soc. 151, 397.

Wynn-Williams, C. G., Becklin, E. E., and Neugebauer, G.: 1974, Astrophys. J. $189,473$. 